\title{
A tetraploidization event shaped the Aquilaria sinensis genome and contributed to the ability of sesquiterpenes synthesis
}

\author{
Fanbo Meng ${ }^{1,2}$, Tianzhe Chu ${ }^{1,3}$, Qiang Tang ${ }^{1,2}$ and Wei Chen ${ }^{1,2,45^{*}}$
}

\begin{abstract}
Background: Agarwood, generated from the Aquilaria sinensis, has high economic and medicinal value. Although its genome has been sequenced, the ploidy of $A$. sinensis paleopolyploid remains unclear. Moreover, the expression changes of genes associated with agarwood formation were not analyzed either.

Results: In the present work, we reanalyzed the genome of $A$. sinensis and found that it experienced a recent tetraploidization event 63-71 million years ago (Mya). The results also demonstrated that the $A$. sinensis genome had suffered extensive gene deletion or relocation after the tetraploidization event, and exhibited accelerated evolutionary rates. At the same time, an alignment of homologous genes related to different events of polyploidization and speciation were generated as well, which provides an important comparative genomics resource for Thymelaeaceae and related families. Interestingly, the expression changes of genes related to sesquiterpene synthesis in wounded stems of $A$. sinensis were also observed. Further analysis demonstrated that polyploidization promotes the functional differentiation of the key genes in the sesquiterpene synthesis pathway.

Conclusions: By reanalyzing its genome, we found that the tetraploidization event shaped the A. sinensis genome and contributed to the ability of sesquiterpenes synthesis. We hope that these results will facilitate our understanding of the evolution of $A$. sinensis and the function of genes involved in agarwood formation.
\end{abstract}

Keywords: Aquilaria sinensis, Tetraploidization, Collinearity, Transcriptome, Sesquiterpenoid biosynthesis

\section{Background}

Aquilaria sinensis $(2 \mathrm{n}=16)$ is the species of the genus Aquilaria of Thymelaeaceae. It grows mainly in tropical climates and is an economically important medical plant. Once wounded or infected by fungi, A. sinensis can produce a precious natural flavor product called agarwood[1], which is widely used in traditional Chinese medicine, handicrafts and religious ceremonies worldwide[1-4]. Due to the limited natural production and

\footnotetext{
* Correspondence: greatchen@ncst.edu.cn

${ }^{1}$ State Key Laboratory of Southwestern Chinese Medicine Resources, Chengdu University of Traditional Chinese Medicine, 611137 Chengdu, China ${ }^{2}$ Innovative Institute of Chinese Medicine and Pharmacy, Chengdu University of Traditional Chinese Medicine, 611137 Chengdu, China

Full list of author information is available at the end of the article
}

increasing demand of agarwood, the $A$. sinensis species are on the verge of extinction. Therefore, clarifying the evolution of $A$. sinensis and the mechanism of agarwood synthesis will be helpful for the protection of $A$. sinensis and will also improve the production of agarwood.

Whole-genome duplication (WGD) or polyploidy, followed by gene loss and diploidization, is an important evolutionary force for many organisms, which not only contributes to their origin and diversification, but also facilitates their adaptation to environmental changes[58]. In particular, recursive polyploidization, polyploidization of different ploidy, and genome rearrangement have been observed in angiosperms $[9,10]$. As a valuable plant, the genome of $A$. sinensis has been sequenced by two independent groups[11, 12]. It was reported that the

(c) The Author(s). 2021 Open Access This article is licensed under a Creative Commons Attribution 4.0 International License, which permits use, sharing, adaptation, distribution and reproduction in any medium or format, as long as you give appropriate credit to the original author(s) and the source, provide a link to the Creative Commons licence, and indicate if changes were made. The images or other third party material in this article are included in the article's Creative Commons licence, unless indicated otherwise in a credit line to the material. If material is not included in the article's Creative Commons licence and your intended use is not permitted by statutory regulation or exceeds the permitted use, you will need to obtain permission directly from the copyright holder. To view a copy of this licence, visit http://creativecommons.org/licenses/by/4.0/. The Creative Commons Public Domain Dedication waiver (http://creativecommons.org/publicdomain/zero/1.0/) applies to the data made available in this article, unless otherwise stated in a credit line to the data. 
genome of $A$. sinensis experienced a recent WGD event[11]. However, due to the lack of in-depth exploration, the ancestral ploidy of $A$. sinensis remains unclear.

Recent studies also demonstrated that agarwood contains many chemical components with different pharmacological activities, such as sesquiterpenes, diterpenes, and chromones[13-16]. Therefore, based on the transcriptome data, the expression profiles of genes related to the sesquiterpene synthesis or stress responses during the formation of agarwood were analyzed[17-19]. Accordingly, the hypothesis that agarwood is the product of plant defense reaction was proposed[17]. Since no reference genome was available then, it was impossible to analyze the genes participated in the entire regulatory pathway of sesquiterpene synthesis. By combining the genome and transcriptome data of $A$. sinensis, the complete set of genes participated in sesquiterpenoid production, plant defense and agarwood production were analyzed in a recent work[12]. It was found that those genes participate in the biosynthesis of sesquiterpenoids via the mevalonic acid (MVA), 1-deoxy-D-xylulose-5-phosphate (DOXP), and methylerythritol phosphate (MEP) pathways. However, the expression changes of genes involved in sesquiterpene biosynthesis during the formation of agarwood have not been analyzed.

Keeping these in mind, in the present work, by using our previously developed pipeline for decoding complex genomes[20, 21], we reanalyzed the $A$. sinensis genome by selecting the grape (Vitis vinifera) and cacao (Theobroma cacao) genomes as the references, and inferred the ploidy of $A$. sinensis paleopolyploid and its occurrence time. By performing the collinearity analysis, an alignment of paralogous and orthologous genes among the three genomes was generated. In order to understand the molecular mechanism of agarwood formation after injury to the stem of $A$. sinensis, the expression changes of genes involved in the sesquiterpene synthesis pathway were analyzed. It was found that the polyploidy events promoted the functional differentiation of sesquiterpene synthesis genes in A. sinensis.

\section{Results}

\section{Homologous Gene Collinearity}

Identification of gene collinearity regions is the basis for deciphering genome structure and elucidating genome evolutionary history. By using ColinearScan[22], we inferred colinear genes within each genome and those between $A$. sinensis and the two reference genomes, namely cacao or grape (Additional file 1: Tables S1 and S2). To identify paralogous genes in colinearity within the $A$. sinensis genome, we found $1,120,155,52$, and 13 syntenic blocks containing at least $4,10,20$, and 50 colinear gene pairs. The total number of gene pairs in these blocks are 8,092, 3,574, 2,223, and 1,084, respectively, with the average of $7.22,23.06,42.75$ and 83.38 gene pairs per block.

The cross-genome homologous blocks, especially those between $A$. sinensis and cacao, are generally longer than those within the $A$. sinensis genome. For example, the genes from $A$. sinensis and cacao form 27,462 colinear gene pairs located in 2,426 homologous blocks with more than four colinear gene pairs, involving 16,525 (56.6\%) and 15,086 (70.3\%) genes from the two genomes, respectively.

\section{Evidence for A Paleo-Tetraploidization Event}

Although a recent whole-genome duplication event has been reported in $A$. sinensis genome [11], the ploidy of its polyploidization remains unclear. To clarify this point, we firstly characterized the synonymous nucleotide substitutions on synonymous substitution sites $\left(K_{S}\right)$ between the above inferred collinear genes. Then, we constructed a dotplot of homologous genes within the A. sinensis genome, in which the collinear genes were displayed by different colors according to their $K_{S}$ values (Fig. 1). If there was no further polyploidization after the shared hexaploidization (i.e. the ECH), each genomic region would have two best matching regions (paralogous, with a smaller median $K_{S}$ ) within the $A$. sinensis genome. However, we found that there is only one best matching region in $A$. sinensis (Fig. 1), indicating that it experienced a tetraploidization. We called it as an $A$. sinensis-specific tetraploidization (AST).

In addition, by plotting the homologous dotplots between $A$. sinensis and grape genomes, we found that there are two best matching regions (orthologous, with a smaller median $K_{S}$ ) in $A$. sinensis, corresponding to each grape chromosome (Additional file 2: Figure S1). For example, for grape chromosome 2, 15, and 16, which are homologous chromosomes produced by ECH[23], each has two best matching regions in the $A$. sinensis genome. Furthermore, we also drew the homologous dotplots between $A$. sinensis and cacao (Additional file 2: Figure $\mathrm{S} 2$ ), which is more closely related to $A$. sinensis than grape and has not been affected by other polyploidizations after the ECH. It was found that each cacao genomic region has two orthologous regions in the $A$. sinensis genome, which is larger than that between grape and $A$. sinensis genomes. These intergenomic analyses demonstrate that $A$. sinensis experienced a tetraploidization event after the $\mathrm{ECH}$ (Fig. 2).

\section{Event-Related Genomic Homology}

According to the above mentioned homologous gene dotplot within the $A$. sinensis genome, we screened the 


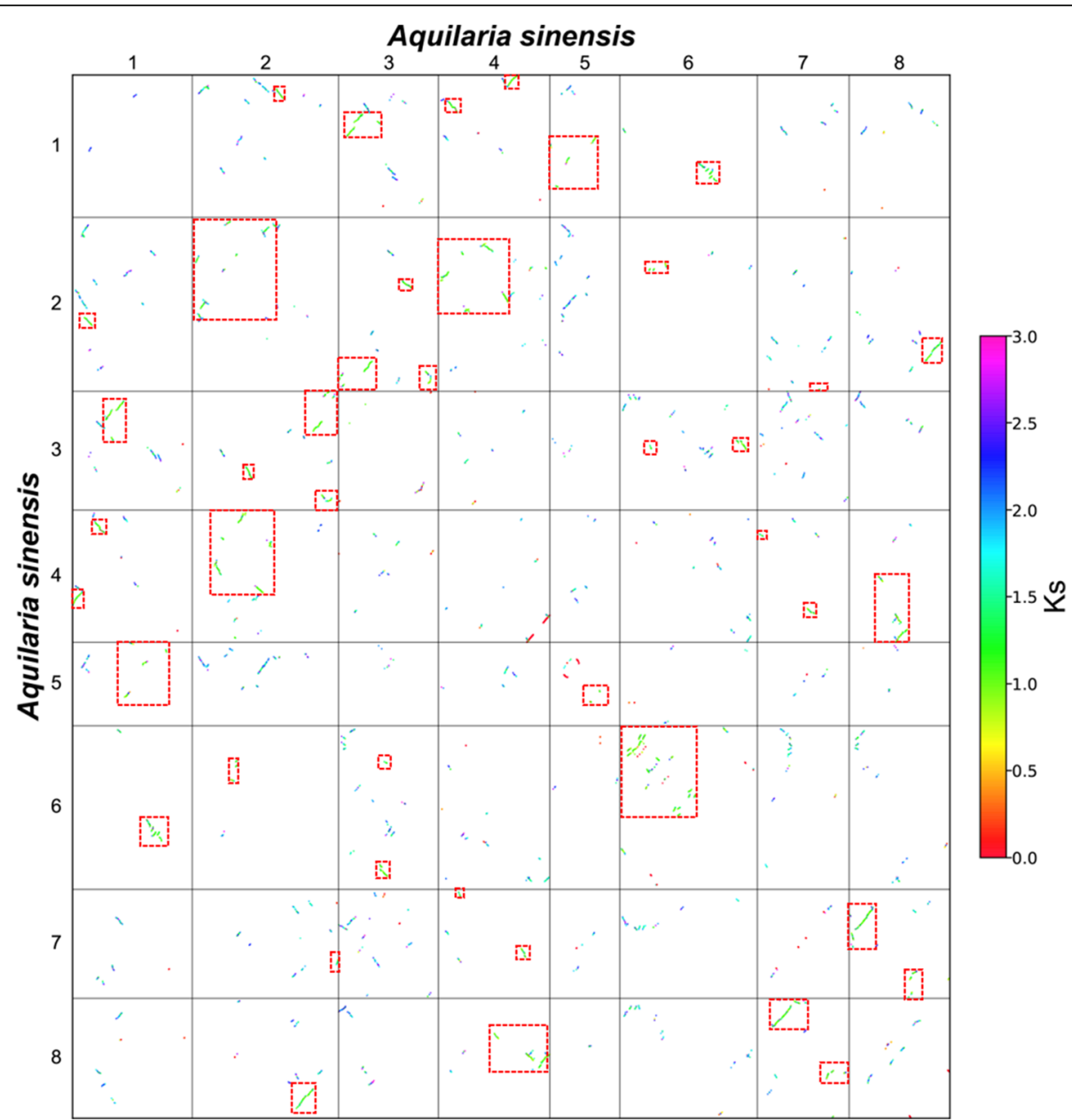

Fig. 1 Homologous Genes Dotplot Within A. sinensis Genome. KS value for homologous genes in each inferred colinear block is shown. Paralogous blocks were identified by red dashed rectangles

blocks with more than 4 gene pairs based on the $K_{S}$ of the collinearity gene pairs and located them to distinguish event-related genome homology regions (Fig. 1). By doing so, the blocks were separated into two types. One type is related to $\mathrm{ECH}$ (with a bigger median $K_{S}$ ) (Additional file 1: Table S3), and the other one related to AST (with a smaller median $K_{S}$ ) (Additional file 1: Table S4). Based on their mutual positions in the dotplot, we divided the $\mathrm{ECH}$-related blocks containing genes from different subgenomes into two groups (Additional file 1: Table S4). Similarly, the blocks in grape and cacao were also filtered and divided into two groups (Additional file 1: Tables S5 and S6). In grape, we found a total of 123 blocks related to the ECH event, forming 2314 paralogous pairs, containing 3,978 genes. In cacao, 3108 paralogous gene pairs containing 4,987 genes in 102 collinear regions were found. In A. sinensis, there are 178 blocks related to the ECH event, forming 1624 gene pairs, containing 2,570 genes, and for the AST event, there are 86 blocks, forming 1,778 gene pairs containing 3,023 genes (Table 1).

Furthermore, we screened the collinearity blocks related to species divergence events between every two genomes (Additional file 1: Table S7), and found that the gene collinearity between genomes is better than that within genomes. For example, 11,201 (38.36\%) genes from A. sinensis could find orthologs in the grape, forming 11,398 orthologous pairs and 486 collinearity blocks.

\section{Multiple Genome Alignment}

By using the grape genome as a reference and filling collinear gene IDs into the polyploid event-related colinear gene table (Additional file 1: Table S8), we performed hierarchical and event-related multiple-genome alignments (grape, cacao, and A. sinensis) and constructed the homologous gene table. The homologous genes from the three species were arranged in 12 columns, including three columns corresponding to paralogous 


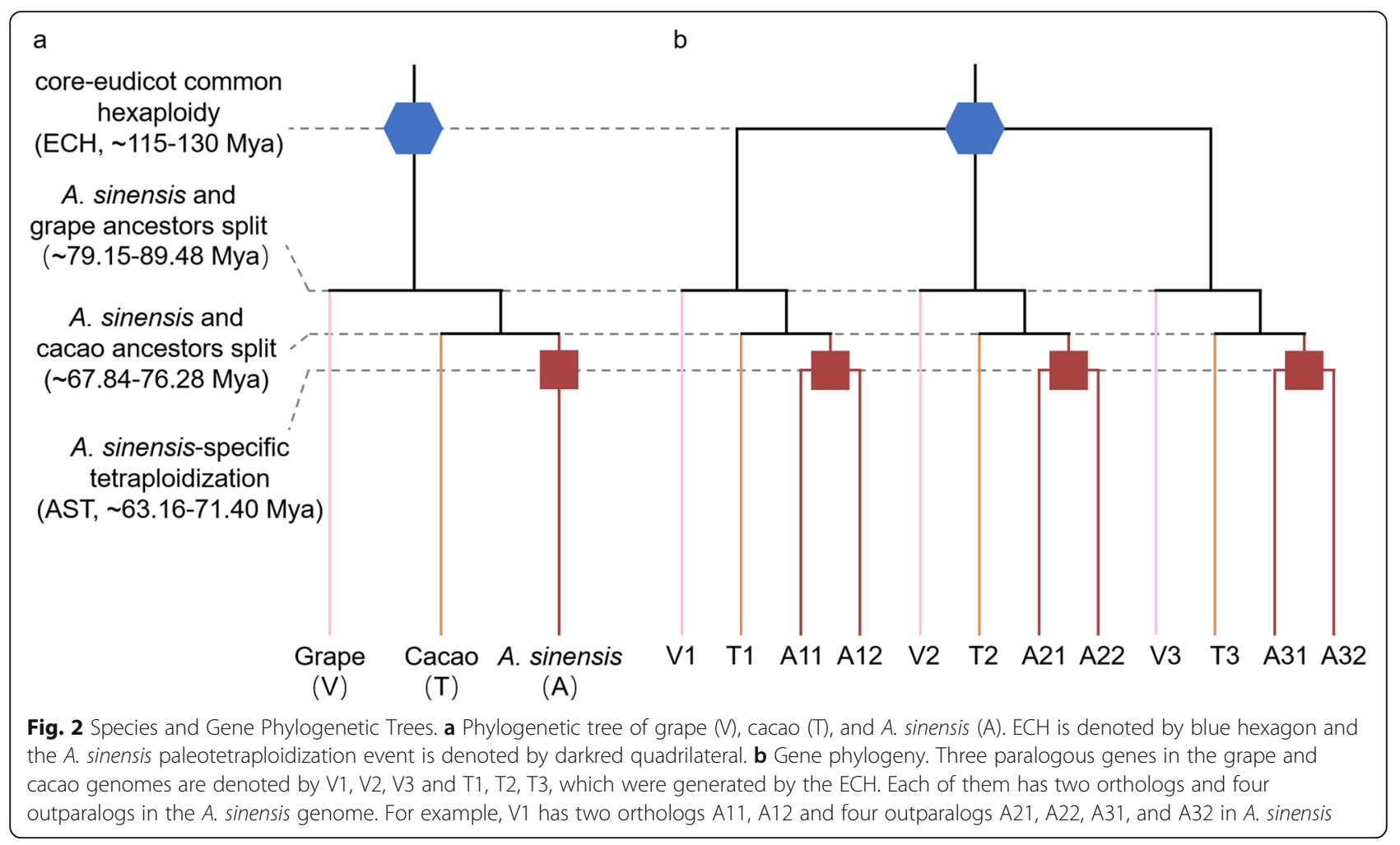

chromosomes of grape, three columns for paralogous chromosomes of cacao, and six columns cumulatively resulting from the ECH followed by AST of A. sinensis. The six $A$. sinensis columns can be classified into three groups corresponding to the hexaploidization-derived chromosomes. Each group contains two columns (corresponding to AST) which is orthologous to a single grape or cacao column, respectively. The table contains paralogous genes in each genome and outparalogs between genomes with each polyploidization, and orthologs between genomes with their ancestral speciation. In order to clearly display the alignment between the three genomes, we converted the homologous collinearity table into a graph (Fig. 3). In Fig. 3, each circle represents a column in the table, and the short lines in the circle represent collinearity genes. In addition, to give a close view of genomic fractionation, a linear graph was

Table 1 Number of Duplicated Genes Within Selected Genomes Related to ECH and AST

\begin{tabular}{lll}
\hline Species & ECH-related $^{\mathbf{a}^{2}}$ & AST -related $^{\mathbf{b}}$ \\
\hline V. vinifera & $123 / 2314 / 3978^{c}$ & - \\
T. cacao & $102 / 3108 / 4987$ & - \\
A. sinensis & $178 / 1624 / 2570$ & $87 / 1778 / 3023$
\end{tabular}

${ }^{a}$ Core eudicot-common hexaploidy (ECH); ${ }^{b}$ Aquilaria-specific tetraploidization; 'numbers of blocks/gene pairs/gene numbers used to display a part of the genome-level alignment (Additional file 2: Figure S3).

Since genes specific to $A$. sinensis and absent from the grape couldn't be represented by using the grape genome as reference, we also constructed a genomic homology table by using cacao as reference (Additional file 1 : Table S9). The table thus obtained supported the paleotetraploidization and represented gene collinearity in $A$. sinensis. For example, when cacao was used as a reference, As1g02078 and As5g01003 were displayed in the table as collinear genes produced by AST. However, they could not be displayed when grape was used as a reference.

\section{Genomic Fractionation}

The above results indicate that many genes were lost and translocated in the A. sinensis genome after experiencing the tetraploidization event. Therefore, we calculated the gene retention or removal rate in the $A$. sinensis genome on each reference chromosome. The chromosomal regions duplicated by the AST often have divergent gene retention levels (Additional file 2: Figures S4 and S5). Regardless of which genome (grape or cacao) was used as the reference, they all showed a lower collinear gene corresponding with $A$. sinensis. In the two sets of orthologous regions, the loss rate of collinear genes relative to different grape chromosomes ranged from 


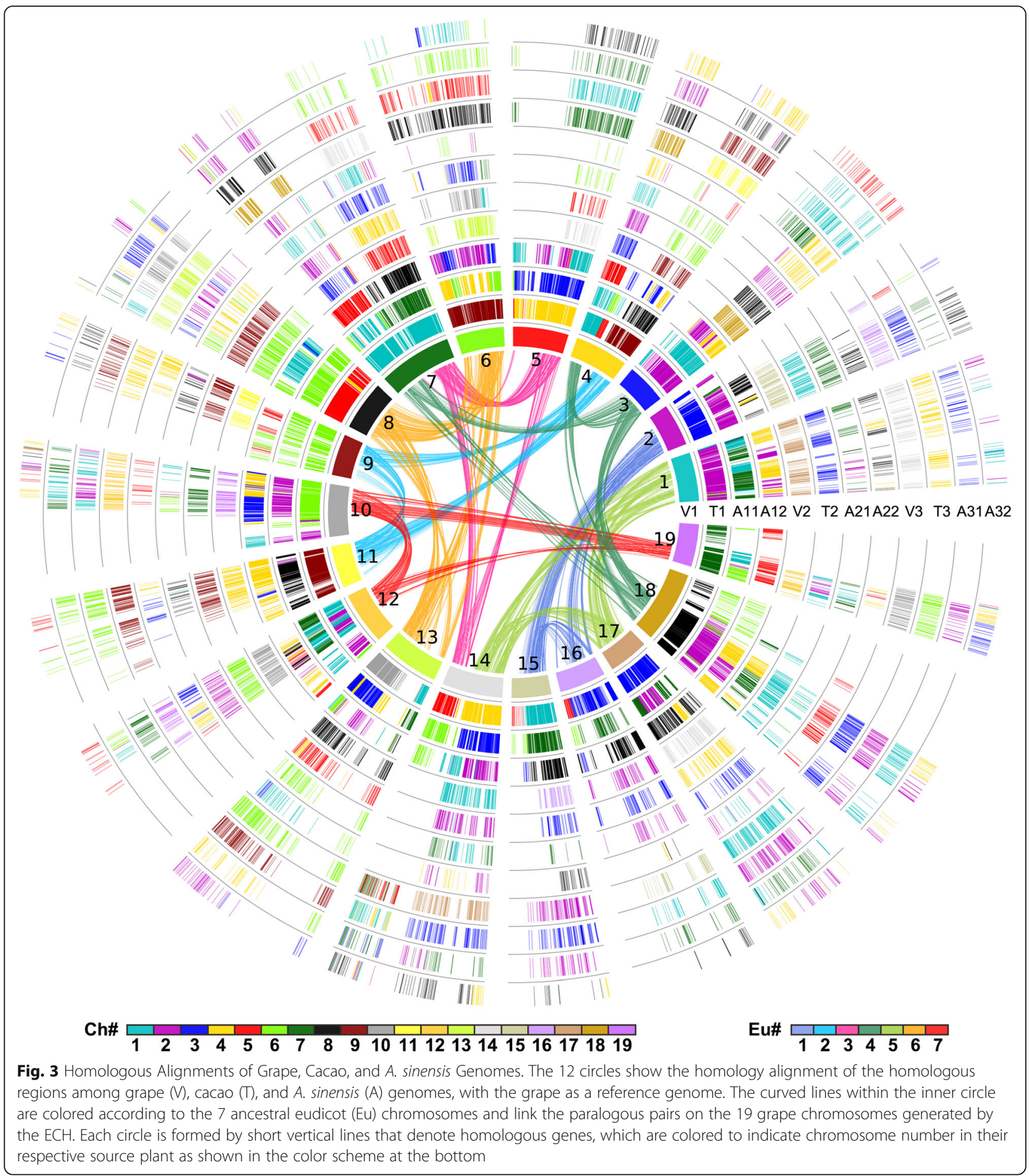

63.80 to $87.52 \%$ (Additional file 1: Table S10). Approximately 81.28 and $87.52 \%$ of the genes in grape chromosome 9 did not have collinear genes in one of the two groups of $A$. sinensis orthologous regions, and $82.93 \%$ of genes did not have correspondence in all homologous regions. The gene loss rates relative to different cacao chromosomes is lower, which ranges from 54.81 to
$80.28 \%$ in each of the two sets of orthologous regions (Additional file 1: Table S11). These results indicate that extensive gene deletion or relocation occurred in the $A$. sinensis genome after the AST event.

Furthermore, by using grape and cacao as reference genomes, we characterized the process of successively removing genes in A. sinensis. Excluding the loss of 
chromosome fragments, the number of removing genes was no greater than 15 consecutive genes in most gene deletion cases. A statistical fitness regression showed that deletion patterns followed a near geometric distribution (Additional file 2: Figures S6 and S7, Additional file 1: Table S12). The extension parameters of geometric distributions were 0.3665 and 0.4201 (goodness of fit F-test $P$-values were 0.8848 and 0.9115 to accept fitness) by using grape and cacao as references, respectively (Additional file 1: Table S13).

\section{Evolutionary Divergence and Dating}

By calculating $K_{S}$ on synonymous nucleotide sites within each genome and among different genomes, we estimated the occurrence time of the AST and other key events. As the $K_{S}$ distribution between paralogs or orthologs is related to specific events, the normal distribution function was used to perform fitting[21, 24, 25]. We statistically determined the location of its mean (or peak) and variance (Fig. 4a, Additional file 2: Figure S8, and Additional file 1: Table S14). It was found that the ECH-related $K_{S}$ peaks from the three genomes are in different positions. The $K_{S}$ of grape is $1.2927(+/-$ $0.1477)$, the $K_{S}$ of cacao is $1.5922(+/-0.1383)$, and the $K_{S}$ of $A$. sinensis is $1.9379(+/-0.3066)$. These values indicate that the evolution rate of grape is the slowest, and the evolution rates of cacao and A. sinensis are $23.17 \%$ ((1.5922-1.2927)/ 1.2927) and 49.91\% ((1.9379-1.2927)/ 1.2927) faster than that of grape, respectively.

In order to adjust the evolution rate corresponding to $\mathrm{ECH}$ events, we aligned the peaks in different genomes to the same position (Fig. 4b, Additional file 1: Table S15) by using the method proposed by Wang et al.[20, 21]. Since the ECH occurred $\sim 115-130$ million years ago (Mya) $[23,26]$, we inferred that the AST event occurred $~ 63.16-71.40$ Mya. In addition, we inferred that ancestors of $A$. sinensis and grape split at $\sim 79.15-89.48$ Mya, and ancestors of $A$. sinensis and cacao split at 67.84-76.28 Mya (Fig. 4c).

\section{Sesquiterpenoid Biosynthesis Related Genes}

In order to explore the copy status of genes related to sesquiterpene synthesis in $A$. sinensis and reference genomes, we used KAAS to annotate genes in the three genomes. Based on the KEGG database, the genes participating in sesquiterpenes synthesis were obtained, including genes in the terpene backbone synthesis pathways (MVA, MEP/DOXP pathway) and the sesquiterpene synthesis. Almost every node in the above mentioned pathway has one or more gene copies among the three species (Fig. 5, Additional file 1: Table S16). A total of 39 genes have been annotated in A. sinensis, including six (-)-germacrene D synthase (GERD) genes. Compared with the MVA pathway (only Acetyl-CoA (ACAT) and diphosphomevalonate decarboxylase (MVD) had the same number of gene copy in three genome), the number of gene copies in the MEP/DOXP pathway (1-deoxyD-xylulose-5-phosphate reductoisomerase (DXR), 2-Cmethyl-D-erythritol 4-phosphate cytidylyltransferase (ISPD), 4-diphosphocytidyl-2-C-methyl-D-erythritol kinase (ISPE), 2-C-methyl-D-erythritol 2,4-cyclodiphosphate synthase (ISPF), and 4-hydroxy-3-methylbut-2en-1-yl diphosphate reductase (ISPH) had the same copy number in A. sinensis, grape and cacao) is relatively stable.

By screening event-related alignments within the species, we obtained the number of sesquiterpene synthesis genes related to different events. In the entire pathway regulating sesquiterpene synthesis of $A$. sinensis, $64.1 \%(25 / 39)$ of genes are related to $\mathrm{ECH}$ event, and $51.3 \%(20 / 39)$ of genes are related to AST event (Additional file 1: Table S17). Especially, there are five 1-deoxy-D-xylulose 5-phosphate synthase (DXS) genes (the rate-limiting enzyme of the MEP/ DOXP pathway [27-29]) in A. sinensis. Genome synteny analysis showed that As3g00334 and As1g01631 fall into two large syntenic blocks on A. sinensis chr3 and chr1 that correspond to a homologous block on grape chr5 and cacao chr4. (Additional file 2: Figure S9), indicating that the As3g00334 and As1g01631 might be a product of the AST event.

By analyzing the transcriptome data of the A. sinensis, we found that the expression of most of genes involved in the regulatory pathways increased after injury, such as As7g01288 (ACAT in MVA pathway), As4g02461 (geranylgeranyl diphosphate synthase (GGPS)) and As4g00176 ((3 S,6E)-nerolidol synthase (NES1)) (Fig. 5, Additional file 1: Table S18). It's interesting that the expression profiles of some homologues of genes are different. For example, the five homologs of the DXS gene have four types of expression profiles, especially the expression of $A s 3 g 00334$ increased after 5 days of injury. In contrast, the expression of As1g01631 remained unchanged.

Furthermore, except for the gene of NAD+dependent farnesol dehydrogenase (FLDH), the expression of sesquiterpene synthesis genes was increased in the injured stems. By constructing a phylogenetic tree of the 30 sesquiterpene synthesis genes in the three species (Additional file 2: Figure S10), we found that only the topological structure of the FLDH gene is consistent with the species phylogeny (Fig. 2a). However, the topological structures formed by the other four types of genes (GERD, NES1, alpha-farnesene synthase (AFS1), and (+)-deltacadinene synthase (CDN1)) were inconsistent with the 

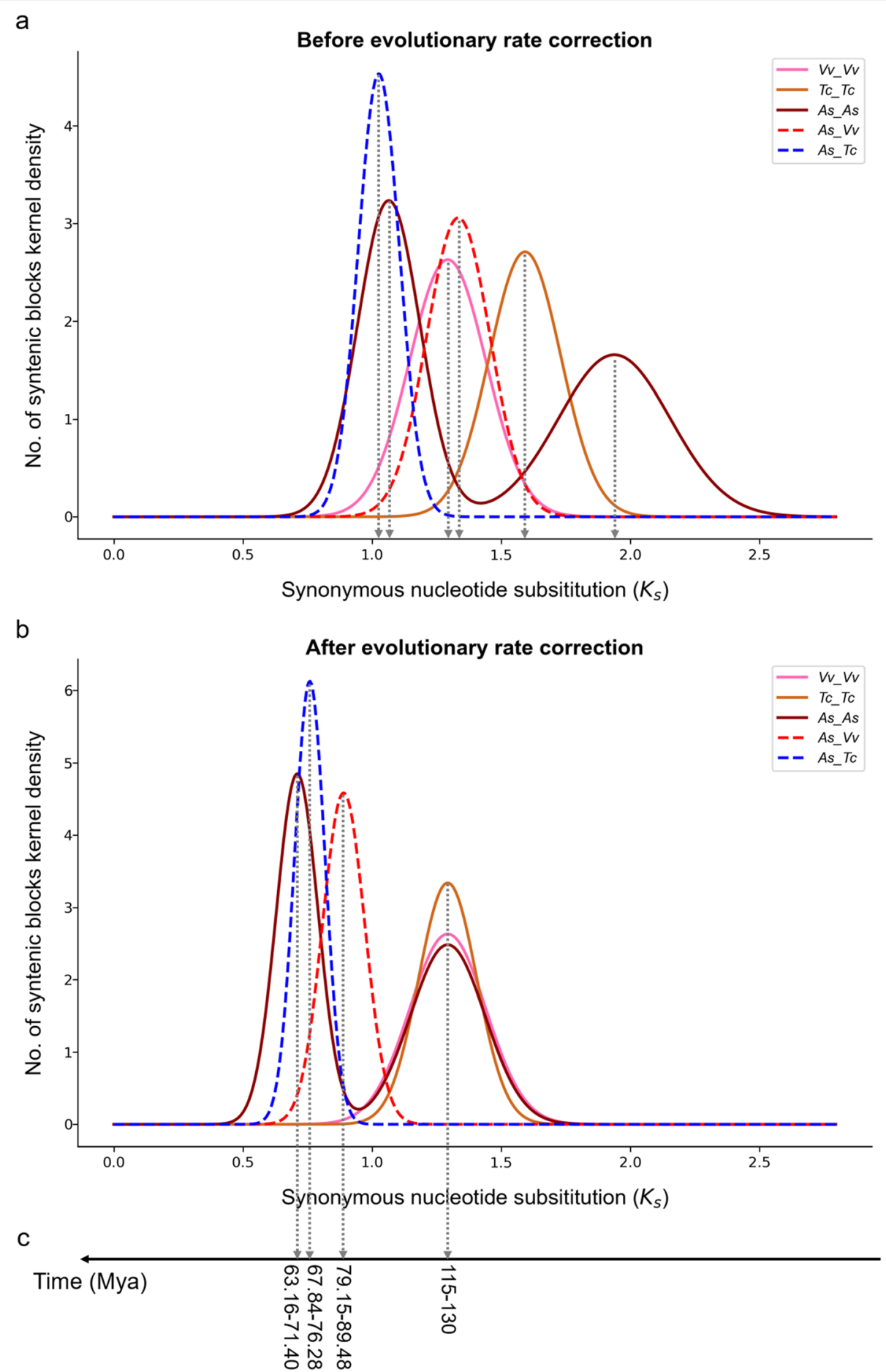

Fig. 4 Dating Evolutionary Events. a Distribution of original median $K_{s}$ between collinear gene pairs in intergenomic blocks (solid curves) and intragenomic blocks (dashed curves). b Distribution of adjusted median $K_{S}$ between collinear gene pairs in intergenomic blocks (solid curves) and intragenomic blocks (dashed curves). c Inferred time

species phylogeny, and all of the A. sinensis genes were located outside of the two reference species, indicating that the evolution rate of these genes in $A$. sinensis is faster than that of grape and cacao genes.
The changes of expression and evolutionary patterns of these genes indicate that they may have specific functions in the process of sesquiterpene synthesis. 


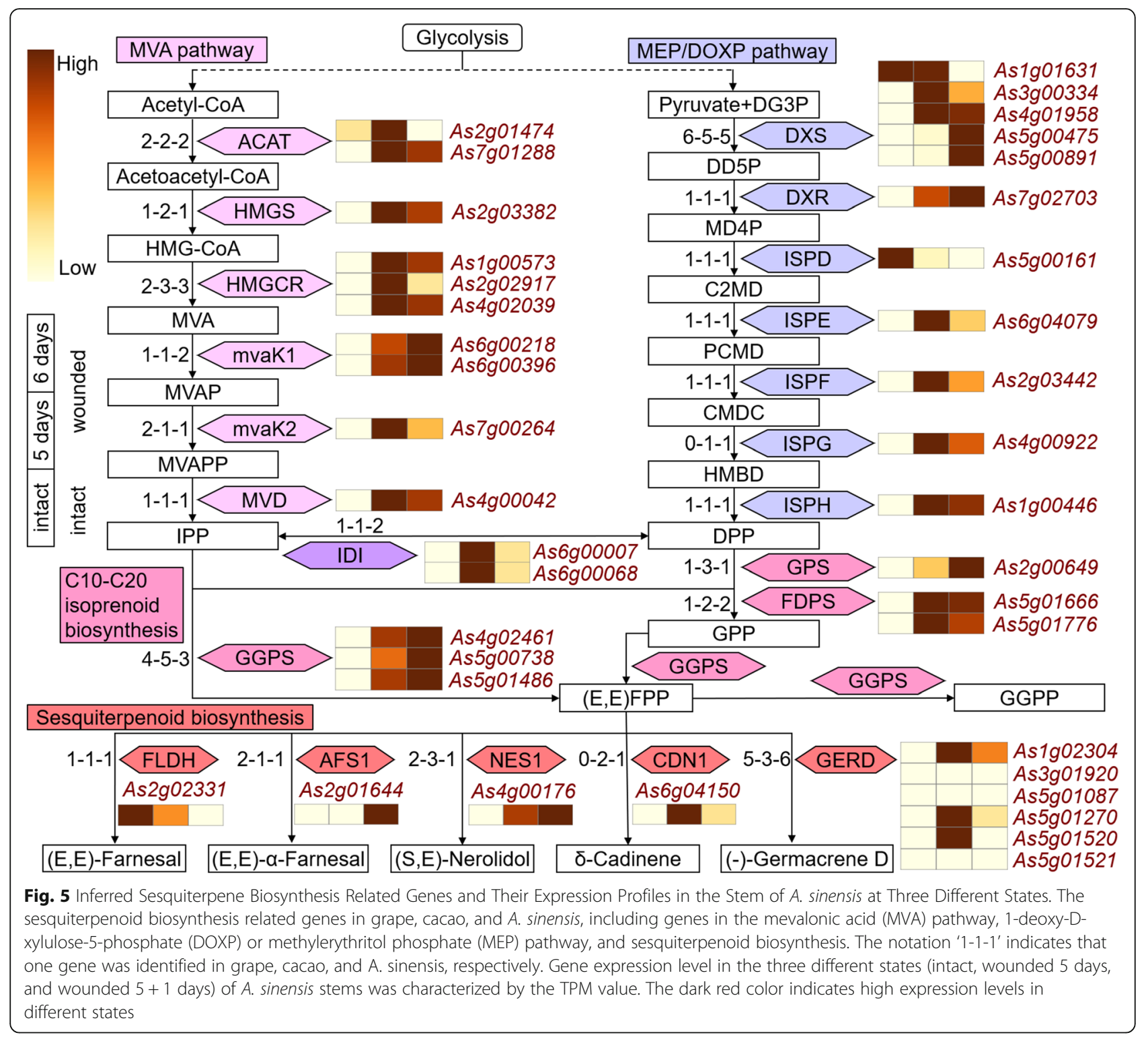

\section{Discussion}

Tetraploidization of $A$. sinensis Genome

Recursive polyploidization, ploidy-different polyploidization, and genome rearrangement events made the structure of plant genomes become complicated [9, 10], which hinders our understanding of the evolution of the genome and the function of genes. Although the genome size could not indicate whether it has experienced polyploidization, the genome sizes of species can be used as a rough estimate for the existence of WGD. The genome size of $A$. sinensis is $\sim 773 \mathrm{Mb}$, which is 72.2 and $75.3 \%$ larger than grape $(\sim 449 \mathrm{Mb})[30]$ and cacao $(\sim$ $441 \mathrm{Mb})[31]$, respectively, indicating that $A$. sinensis have experienced a tetraploidization. A crucial consideration for exploring the multiples of genome-wide polyploidization is to determine the scales of orthologous and paralogous collinear blocks through inter-genome and intra-genome comparisons, respectively. Based on the analysis of the 4DTv distribution of gene pairs in the collinear block, a large accumulation of gene duplications in the $A$. sinensis genome was reported[11]. Ding et al. concluded that the genome of $A$. sinensis seems to have experienced a recent whole-genome duplication event, without any evidence of the ploidy of polyploidization. In the current study, we found the ratios of orthologous regions of grape and cacao with $A$. sinensis are both 1:2, indicating that the polyploidization of $A$. sinensis is a tetraploidization.

In addition, we also generated the gene-colinearity supported homologous (orthologous and paralogous) gene lists, which are helpful to reveal the evolutionary and function-innovation trajectories of genes, gene 
families, regulatory pathways, and other important traits. For example, based on the lists, we found that genes related to the sesquiterpene biosynthetic pathway are expanded during polyploidization events.

\section{Evolutionary Rates}

Different species have different evolutionary rates, the differences may be even greater especially after being affected by polyploidization. To perform authentic dating, we corrected the evolutionary rate of cacao and A. sinensis based on the grape, which has the slowest evolutionary rate. We found that the evolutionary rate of $A$. sinensis is 49.9 and $21.7 \%((1.9379-1.5922) / 1.5922)$ faster than that of grape and cacao, respectively.

\section{Possible Factors of Sesquiterpene Synthesis in A. sinensis}

Previous studies have demonstrated that sesquiterpenes and phenylethyl chromone derivatives are the main compounds in agarwood[32-34], and the terpenoid metabolism pathway is very clear. Polyploidization may promote the evolution of key traits, such as nodulation[35], cotton fiber[26], and $\mathrm{VC}$ biosynthesis[36]. In the present study, we found that the genes of $A$. sinensis involved in the sesquiterpene synthesis pathway were also affected by two polyploidization events. Besides, more than half of the identified genes related to sesquiterpene synthesis have experienced AST events. The function of some sesquiterpene synthesis genes related to AST may be different and play distinct roles in the process of sesquiterpene synthesis. Phylogenetic analysis showed that four of the five types of sesquiterpene synthase genes in A. sinensis evolved faster than those in the other two species, suggesting their distinct functions in $A$. sinensis. In addition, the expression of genes in the sesquiterpene synthesis regulatory pathway has changed after the injury of $A$. sinensis, and most of the genes are highly expressed in the stem. In particular, two genes (As3g00334 and As1g01631) presented opposite expression patterns, indicating that they may have different functions after the AST event.

\section{Conclusions}

In conclusion, by reanalyzing its genome, we found that A. sinensis had experienced a tetraploidization event at $\sim 63-71$ Mya. Further analysis demonstrated that the polyploidization of $A$. sinensis contributed to the functional differentiation of genes related to sesquiterpene synthesis. The present work also provided an alignment of orthologous and paralogous genes related to different events of polyploidization and speciation, which provides an important comparative genomics resource for Thymelaeaceae and related families. We hope that these results will not only be helpful for illustrating the evolution of $A$. sinensis, but also for understanding the functions of genes related to the formation of agarwood.

\section{Methods \\ Genomes and RNA-seq data}

Since the grape and cacao didn't experience recent whole-genome duplication after the core-eudicotcommon hexaploidy $(\mathrm{ECH})$, they were selected as reference genomes in the present work. The genomes of grape, cacao and $A$. sinensis together with their annotation files were downloaded from the public databases listed in Table S19. The RNA-seq data of $A$. sinensis stem (intact, wounded 5 days, and wounded $5+1$ days) generated by Nong et al. was obtained from the NCBI Short Read Archive (SRR10143145, SRR10143137, and SRR10143136)[12].

\section{Gene Collinearity}

The homologous proteins within and between genomes were identified by using the BLASTP[37] (E value $1 \times$ $10^{-5}$ ), and the syntenic blocks consisting of homologous genes were scanned by using the ColinearScan[22]. The maximum gap length between genes that are collinearity along a chromosome was set as 50 genes apart [21, 38]. By using the wgdi package of Python (https://github. com/SunPengChuan/wgdi), we produced dotplots of the homologous gene within or between genomes. All procedures followed the gold-standard pipeline for deciphering complex genomes[20].

\section{Nucleotide Substitution}

Synonymous nucleotide substitutions on synonymous sites $\left(K_{S}\right)$ between colinear homologous genes were estimated by using the YN00 program in the PAML (v4.9i) package with the Nei-Gojobori approach[39]. According to their $K_{S}$ values, we displayed the collinearity gene pairs in the dotplot with different colors, which facilitate to cluster the blocks generated by different events into groups.

\section{Construction of An Event-Related, Colinear Gene Table}

To construct the polyploid event-related colinear gene table with the grape genome as a reference, we listed all grape genes in the first column. Each grape gene may have two extra colinear genes due to the hexaploidy of the genome. Thus, we assigned the extra colinear genes into the other two columns in the table. For each gene from the grape, when there was a corresponding colinear gene in an expected location, we entered its ID in a cell of the corresponding column in the table. When a colinear gene was missing, often due to gene loss or translocation in the genome, we entered a dot in the cell. For the cacao genome, except for sharing hexaploidization with grape, it has no extra duplications. Therefore, we 
assigned one column next to the columns of grape. Meanwhile, for the $A$. sinensis genome that experienced a paleo-tetraploidization event (ref. the analysis in the Result section), we assigned two columns in the table. Finally, a table with 12 columns was built, which reflects layers and layers of threefold and then twofold homology due to recursive polyploidies across the genomes. The cacao-based table was constructed similarly.

\section{Kernel Function Analysis of $K_{s}$}

To exhibit the enrichment of $K_{S}$, we performed a kernel function analysis of the $K_{S}$ distribution of collinear genes within a genome or between genomes. The Gaussian_kde function in the scipy package of Python was used to estimate the probability density of each $K_{S}$ list, and to obtain the density distribution curve. Subsequently, the curve_fit function was used to obtain the proper curves by adjusting related parameters with the Gaussian equation. We adjusted the $\mathrm{R}^{2}$ to be as close to 1 as possible, and finally determined the goodness of the fit.

\section{KEGG Annotation}

Assigning KEGG Orthologies (KO) to the predicted proteins of the three genomes and generating the KEGG metabolic pathways were done with KAAS (KEGG Automatic Annotation Server)[40] using the bidirectional best hit method.

\section{Calculation of Gene Expression Levels}

The raw Illumina reads were trimmed by using Trimmomatic (v0.39)[41]. The FastQC[42] qualified reads were aligned to the $A$. sinensis genome by using the hierarchical indexing for spliced alignment of transcripts (HISA T2)[43]. The aligned results were sorted and transferred the format from 'bam' to 'sam' by using SAMtools[44], and the transcripts per kilobase of exon model per million mapped reads (TPM) for each $A$. sinensis gene model was calculated by using Stringtie [45].

\section{Construction of Phylogenetic Tree}

The peptide sequences (PEP) of the sesquiterpenoid biosynthesis genes in three genomes were aligned by using ClustalW[46, 47]. The tree construction was performed by using the neighboring-joining approach in MEGA $\mathrm{X}[48]$ with default parameters. The reliability of an inferred tree was characterized with bootstrap analysis with 1000 replications.

\section{Abbreviations}

WGD: Whole-genome duplication; As:Aquilaria sinensis; Vv: Vitis vinifera; Tc: Theobroma cacao; MVA: Mevalonic acid; DOXP: 1-deoxy-D-xylulose-5phosphate; MEP: Methylerythritol phosphate; ECH: Core Eudicot-Common Hexaploidy; Mya: Million years ago; $K_{s}$ : Synonymous nucleotide substitutions on synonymous sites; AST: A. sinensis-specific tetraploidization; ACAT: AcetylCoA C-acetyltransferase; HMGS: Hydroxymethylglutaryl-CoA synthase; HNGC
R: Hydroxymethylglutaryl-CoA reductase; mvaK1: Mevalonate kinase; mvaK2: Phosphomevalonate kinase; MVD: Diphosphomevalonate decarboxylase; DXS: 1-deoxy-D-xylulose-5-phosphate synthase; DXR: 1-deoxyD-xylulose-5-phosphate reductoisomerase; ISPD: 2-C-methyl-D-erythritol 4phosphate cytidylyltransferase; ISPE: 4-diphosphocytidyl-2-C-methyl-Derythritol kinase; ISPF: 2-C-methyl-D-erythritol 2,4-cyclodiphosphate synthase; ISPG: (E)-4-hydroxy-3-methylbut-2-enyl-diphosphate synthase; ISPH: 4hydroxy-3-methylbut-2-en-1-yl diphosphate reductase; IDI: Isopentenyldiphosphate Delta-isomerase; GPS: Geranyl diphosphate synthase; FDPS: Farnesyl diphosphate synthase; GGPS: Geranylgeranyl diphosphate synthase, type III; FLDH: NAD+-dependent farnesol dehydrogenase; AFS1: Alpha-farnesene synthase; NES1: (3 S,6E)-nerolidol synthase; CDN1: (+)delta-cadinene synthase; GERD: (-)-germacrene D synthase

\section{Supplementary Information}

The online version contains supplementary material available at https://doi. org/10.1186/s12864-021-07965-9.

Additional file 1: Table S1. Number of Homologous Blocks and Gene Pairs Within a Genome or Between Genomes. Table S2. Number of Homologous Genes Within a Genome or Between Genomes. Table S3. Information about the Blocks Related to ECH Events in A. sinensis genome. Table S4. Information about the Blocks Related to AST Events in A. sinensis genome. Table S5. Information about the Blocks Related to ECH Events in Grape Genome. Table S6. Information about the Blocks Related to ECH Events in Cacao Genome. Table S7. Number of Collinearity Blocks, Gene Pairs, and Genes Related to Species Divergence Events Between Genomes. Table S8. Multiple Genome Alignment with Grape as Reference. Table S9. Genome Alignment Between Cacao and A.sinensis.Table S10. A. sinensis Gene Loss Rates and Gene Translocation with Grape as Reference Genome. Table S11. A. sinensis Gene Loss Rates and Gene Translocation with Cacao as Reference Genome. Table S12. Gene Loss in A. sinensis Using Grape and Cacao as References. Table S13. The Observed Distribution of Gene Loss and Translocation Numbers Fitted by Using Different Density Curves of Geometry Distribution. Table S14. Kernel Function Analysis of $K_{S}$ Distribution Related to Duplication Events Within each Genome and Between Genomes (before Evolutionary Rate Correction). Table S15. Kernel Function Analysis of $K_{S}$ Distribution Related to Duplication Events Within each Genome and Between Genomes (after Evolutionary Rate Correction). Table S16. The Information of Terpenoid Backbone Biosynthesis and Sesquiterpenoid Biosynthesis Genes in the Three Genomes by KEGG. Table S17. Sesquiterpenoid Biosynthesis Genes. Table S18. The Expression Values (TPM) of $A$. sinensis stems in Three States (intact, wounded 5 days and wounded $5+1$ days) by RNA-sEq. Table S19. Genomic Data Information.

Additional file 2: Figure S1. Homologous Genes Dotplot between A. sinensis and Grape Genomes. Figure S2. Homologous Genes Dotplot between A. sinensis and Cacao Genomes. Figure S3. Local Homologous Alignments of Grape, Cacao, and A. sinensis Genomes. Figure S4. The Retention of Duplicated Genes Residing in Two Subgenomes of $A$. sinensis using the Grape as Reference. Figure S5. The Retention of Duplicated Genes Residing in Two Subgenomes of A. sinensis using the Cacao as Reference. Figure S6. Near Geometric Distribution of Continually Lost or Translocated Genes between A. sinensis and Grape. Figure S7. Near Geometric Distribution of Continually Lost or Translocated Genes between A. sinensis and Cacao. Figure S8. Histograms and Gaussian Fitted Curves of $K_{S}$ between Colinear Homologous Genes. Figure S9. Gene Synteny Analysis among Grape, Cacao, and A. sinensis. Figure S10. The Phylogenetic Tree Constructed using Sesquiterpene Synthesis Genes from Grape, Cacao, and A. sinensis.

Acknowledgements

We appreciate the anonymous reviewers for their constructive comments.

\section{Authors' contributions}

WC conceived and led the research. FM performed the analysis. TC, QT contributed to data collection and joined constructive discussions. FM and WC wrote the paper. All authors read and approved the final manuscript. 


\section{Funding}

We appreciate financial support from the National Nature Science Foundation of China (No.31771471).

\section{Availability of data and materials}

All data generated or analysed during this study were included in this published article and the Additional files.

\section{Declarations}

\section{Ethics approval and consent to participate}

Not applicable.

\section{Consent for publication}

Not applicable.

\section{Competing interests}

The authors declare that they have no competing interests.

\begin{abstract}
Author details
${ }^{1}$ State Key Laboratory of Southwestern Chinese Medicine Resources, Chengdu University of Traditional Chinese Medicine, 611137 Chengdu, China. ${ }^{2}$ Innovative Institute of Chinese Medicine and Pharmacy, Chengdu University of Traditional Chinese Medicine, 611137 Chengdu, China. ${ }^{3}$ School of Pharmacy, Chengdu University of Traditional Chinese Medicine, 611137 Chengdu, China. ${ }^{4}$ School of Basic Medical Sciences, Chengdu University of Traditional Chinese Medicine, 611137 Chengdu, China. ${ }^{5}$ School of Life Sciences, North China University of Science and Technology, 063210 Tangshan, China.
\end{abstract}

Received: 2 June 2021 Accepted: 25 August 2021 Published online: 07 September 2021

\section{References}

1. Chhipa H, Chowdhary K, Kaushik N: Artificial production of agarwood oil in Aquilaria sp. by fungi: a review. Phytochemistry Reviews 2017, 16:1-26.

2. Persoon G, Beek H: Growing 'The Wood of The Gods': Agarwood Production in Southeast Asia. In., vol. 5; 2008: 245-262.

3. Okudera Y, Ito MJPB: Production of agarwood fragrant constituents in Aquilaria calli and cell suspension cultures. 2009, 26(3):307-315.

4. Kakino M, Tazawa S, Maruyama H, Tsuruma K, Araki Y, Shimazawa M, Hara H: Laxative effects of agarwood on low-fiber diet-induced constipation in rats. BMC Complement Altern Med 2010, 10:68-68.

5. Jiao Y, Wickett NJ, Ayyampalayam S, Chanderbali AS, Landherr L, Ralph PE, Tomsho LP, Hu Y, Liang H, Soltis PS et al: Ancestral polyploidy in seed plants and angiosperms. Nature 2011, 473(7345):97-100.

6. Van de Peer Y, Maere S, Meyer A: The evolutionary significance of ancient genome duplications. Nature reviews Genetics 2009, 10(10):725-732.

7. Soltis DE, Bell CD, Kim S, Soltis PS: Origin and early evolution of angiosperms. Annals of the New York Academy of Sciences 2008, 1133:325

8. Zhang L, Wu S, Chang X, Wang X, Zhao Y, Xia Y, Trigiano RN, Jiao Y, Chen F: The ancient wave of polyploidization events in flowering plants and their facilitated adaptation to environmental stress. Plant, cell \& environment 2020, 43(12):2847-2856.

9. Soltis PS, Marchant DB, Van de Peer Y, Soltis DE: Polyploidy and genome evolution in plants. Current opinion in genetics \& development 2015, 35: $119-125$.

10. Sankoff $D$, Zheng C: Fractionation, rearrangement and subgenome dominance. Bioinformatics 2012, 28(18):i402-i408.

11. Ding X, Mei W, Lin Q, Wang H, Wang J, Peng S, Li H, Zhu J, Li W, Wang P et al: Genome sequence of the agarwood tree Aquilaria sinensis (Lour.) Spreng: the first chromosome-level draft genome in the Thymelaeceae family. Gigascience 2020, 9(3): giaa013.

12. Nong W, Law STS, Wong AYP, Baril T, Swale T, Chu LM, Hayward A, Lau DTW, Hui JHL: Chromosomal-level reference genome of the incense tree Aquilaria sinensis. Mol Ecol Resour 2020, 20(4):971-979.

13. Gao M, Han X, Sun Y, Chen H, Yang Y, Liu Y, Meng H, Gao Z, Xu Y, Zhang Z et al: Overview of sesquiterpenes and chromones of agarwood originating from four main species of the genus Aquilaria. RSC Advances 2019, 9(8): 4113-4130.
14. Alfinda Novi K, Mulyadi T, Nanik Siti A: Review: Secondary Metabolites of Aquilaria, a Thymelaeaceae Genus. Mini-Reviews in Organic Chemistry 2018, 15(1):36-55

15. Wang S, Yu Z, Wang C, Wu C, Guo P, Wei J: Chemical Constituents and Pharmacological Activity of Agarwood and Aquilaria Plants. 2018, 23(2):342.

16. He Q, Hu DB, Zhang L, Xia MY, Yan H, Li XN, Luo JF, Wang YS, Yang JH, Wang $\mathrm{YH}$ : Neuroprotective compounds from the resinous heartwood of Aquilaria sinensis. Phytochemistry 2021, 181:112554.

17. Xu Y, Zhang Z, Wang M, Wei J, Chen H, Gao Z, Sui C, Luo H, Zhang X, Yang $Y$ et al: Identification of genes related to agarwood formation: transcriptome analysis of healthy and wounded tissues of Aquilaria sinensis. BMC Genomics 2013, 14:227.

18. Wang X, Gao B, Liu X, Dong X, Zhang Z, Fan H, Zhang L, Wang J, Shi S, Tu $P$ : Salinity stress induces the production of 2-(2-phenylethyl)chromones and regulates novel classes of responsive genes involved in signal transduction in Aquilaria sinensis calli. BMC plant biology 2016, 16(1):119.

19. Wang X, Zhang Z, Dong X, Feng Y, Liu X, Gao B, Wang J, Zhang L, Wang J, Shi $S$ et al: Identification and functional characterization of three type III polyketide synthases from Aquilaria sinensis calli. Biochemical and biophysical research communications 2017, 486(4):1040-1047.

20. Wang J, Sun P, Li Y, Liu Y, Yang N, Yu J, Ma X, Sun S, Xia R, Liu X et al: An Overlooked Paleotetraploidization in Cucurbitaceae. Mol Biol Evol 2018, 35(1):16-26.

21. Wang J, Yuan J, Yu J, Meng F, Sun P, Li Y, Yang N, Wang Z, Pan Y, Ge W et al: Recursive Paleohexaploidization Shaped the Durian Genome. Plant physiology 2019, 179(1):209-219.

22. Wang X, Shi X, Li Z, Zhu Q, Kong L, Tang W, Ge S, Luo J: Statistical inference of chromosomal homology based on gene colinearity and applications to Arabidopsis and rice. BMC Bioinformatics 2006, 7:447-447.

23. Jaillon O, Aury JM, Noel B, Policriti A, Clepet C, Casagrande A, Choisne N, Aubourg S, Vitulo N, Jubin C et al: The grapevine genome sequence suggests ancestral hexaploidization in major angiosperm phyla. Nature 2007, 449(7161):463-467.

24. Yang $Y$, Sun $P$, Lv L, Wang D, Ru D, Li Y, Ma T, Zhang L, Shen X, Meng F et al: Prickly waterlily and rigid hornwort genomes shed light on early angiosperm evolution. Nature plants 2020, 6(3):215-222.

25. Huang G, Wu Z, Percy RG, Bai M, Li Y, Frelichowski JE, Hu J, Wang K, Yu JZ, Zhu Y: Genome sequence of Gossypium herbaceum and genome updates of Gossypium arboreum and Gossypium hirsutum provide insights into cotton A-genome evolution. Nature genetics 2020, 52(5):516-524.

26. Paterson $\mathrm{AH}$, Wendel JF, Gundlach $\mathrm{H}$, Guo H, Jenkins J, Jin D, Llewellyn D, Showmaker KC, Shu S, Udall J et al: Repeated polyploidization of Gossypium genomes and the evolution of spinnable cotton fibres. Nature 2012, 492(7429):423-427.

27. Mandel MA, Feldmann KA, Herrera-Estrella L, Rocha-Sosa M, León P: CLA1, a novel gene required for chloroplast development, is highly conserved in evolution. The Plant journal: for cell and molecular biology 1996, 9(5):649-658.

28. Estévez JM, Cantero A, Romero C, Kawaide H, Jiménez LF, Kuzuyama T, Seto $H$, Kamiya $Y$, León P: Analysis of the expression of CLA1, a gene that encodes the 1-deoxyxylulose 5-phosphate synthase of the 2-C-methyl-Derythritol-4-phosphate pathway in Arabidopsis. Plant physiology 2000 124(1):95-104.

29. Estévez JM, Cantero A, Reindl A, Reichler S, León P: 1-Deoxy-d-xylulose-5phosphate Synthase, a Limiting Enzyme for Plastidic Isoprenoid Biosynthesis in Plants*. Journal of Biological Chemistry 2001, 276(25):22901-22909.

30. Massonnet M, Cochetel N, Minio A, Vondras AM, Lin J, Muyle A, Garcia JF, Zhou Y, Delledonne M, Riaz S et al: The genetic basis of sex determination in grapes. Nat Commun 2020, 11(1):2902.

31. Argout X, Martin G, Droc G, Fouet O, Labadie K, Rivals E, Aury JM, Lanaud C: The cacao Criollo genome v2.0: an improved version of the genome for genetic and functional genomic studies. BMC Genomics 2017, 18(1):730.

32. Hashimoto K, Nakahara S, Inoue T, Sumida Y, Takahashi M, Masada Y: A New Chromone from Agarwood and Pyrolysis Products of Chromone Derivatives. CHEMICAL \& PHARMACEUTICAL BULLETIN 1985, 33(11):5088-5091.

33. Ishihara M, Tsuneya T, Uneyama K: Fragrant sesquiterpenes from agarwood. Phytochemistry 1993, 33(5):1147-1155.

34. Kumeta $Y$, Ito M: Characterization of delta-guaiene synthases from cultured cells of Aquilaria, responsible for the formation of the sesquiterpenes in agarwood. Plant Physiol 2010, 154(4):1998-2007.

35. Wang J, Sun P, Li Y, Liu Y, Yu J, Ma X, Sun S, Yang N, Xia R, Lei T et al: Hierarchically Aligning 10 Legume Genomes Establishes a Family-Level Genomics Platform. Plant Physiol 2017, 174(1):284-300. 
36. Wang JP, Yu JG, Li J, Sun PC, Wang L, Yuan JQ, Meng FB, Sun SR, Li YX, Lei TY et al: Two Likely Auto-Tetraploidization Events Shaped Kiwifruit Genome and Contributed to Establishment of the Actinidiaceae Family. iScience 2018, 7:230-240

37. Camacho C, Coulouris G, Avagyan V, Ma N, Papadopoulos J, Bealer K, Madden TL: BLAST+: architecture and applications. BMC Bioinformatics 2009, 10:421-421.

38. Wang $X$, Guo H, Wang J, Lei T, Liu T, Wang Z, Li Y, Lee TH, Li J, Tang H, et al. Comparative genomic de-convolution of the cotton genome revealed a decaploid ancestor and widespread chromosomal fractionation. New Phytol. 2016;209(3):1252-63.

39. Yang Z. PAML 4: phylogenetic analysis by maximum likelihood. Mol Biol Evol. 2007;24(8):1586-91.

40. Moriya Y, Itoh M, Okuda S, Yoshizawa AC, Kanehisa M. KAAS: an automatic genome annotation and pathway reconstruction server. Nucleic Acids Res. 2007;35(Web Server issue):W182-185.

41. Bolger AM, Lohse M, Usadel B. Trimmomatic: a flexible trimmer for Illumina sequence data. Bioinformatics. 2014;30(15):2114-20.

42. Andrews S: FASTQC. A quality control tool for high throughput sequence data. $\vee$ 0.11.9. http://www.bioinformatics.babraham.ac.uk/projects/fastqc In.; 2010.

43. Kim D, Langmead B, Salzberg SL: HISAT: a fast spliced aligner with low memory requirements. Nat Methods 2015, 12(4):357-360.

44. Li H, Handsaker B, Wysoker A, Fennell T, Ruan J, Homer N, Marth G, Abecasis G, Durbin R, Genome Project Data Processing S: The Sequence Alignment/ Map format and SAMtools. Bioinformatics 2009, 25(16):2078-2079.

45. Pertea M, Pertea GM, Antonescu CM, Chang TC, Mendell JT, Salzberg SL: StringTie enables improved reconstruction of a transcriptome from RNA-seq reads. Nat Biotechnol 2015, 33(3):290-295.

46. Thompson JD, Higgins DG, Gibson TJ: CLUSTAL W: improving the sensitivity of progressive multiple sequence alignment through sequence weighting, position-specific gap penalties and weight matrix choice. Nucleic acids research 1994, 22(22):4673-4680.

47. Larkin MA, Blackshields G, Brown NP, Chenna R, McGettigan PA, McWilliam H, Valentin F, Wallace IM, Wilm A, Lopez R et al: Clustal W and Clustal X version 2.0. Bioinformatics 2007, 23(21):2947-2948.

48. Kumar S, Stecher G, Li M, Knyaz C, Tamura K: MEGA X: Molecular Evolutionary Genetics Analysis across Computing Platforms. Mol Biol Evol 2018, 35(6):1547-1549.

\section{Publisher's Note}

Springer Nature remains neutral with regard to jurisdictional claims in published maps and institutional affiliations.

\section{Ready to submit your research? Choose BMC and benefit from:}

- fast, convenient online submission

- thorough peer review by experienced researchers in your field

- rapid publication on acceptance

- support for research data, including large and complex data types

- gold Open Access which fosters wider collaboration and increased citations

- maximum visibility for your research: over $100 \mathrm{M}$ website views per year

At BMC, research is always in progress.

Learn more biomedcentral.com/submissions 